\title{
Assessing the Effect of Spacecraft Motion on Single-Spacecraft Solar Wind Tracking Techniques
}

\author{
T.M. Conlon • S.E. Milan · J.A. Davies
}

Received: 25 November 2013 / Accepted: 30 April 2014 / Published online: 17 May 2014

(C) The Author(s) 2014. This article is published with open access at Springerlink.com

\begin{abstract}
Recent advances in wide-angle imaging by the Solar Mass Ejection Imager (SMEI) on board the Coriolis spacecraft and more recently by the Heliospheric Imagers (HI) aboard NASA's Solar TErrestrial RElations Observatory (STEREO), have enabled solar wind transients to be imaged and tracked from the Sun to 1 AU and beyond. In this paper we consider two of the techniques that have been used to determine the propagation characteristics of solar wind transients based on single-spacecraft observations, in particular propagation direction and radial speed. These techniques usually assume that the observing spacecraft remains stationary for the duration of observation of the solar wind transient. We determine the inaccuracy introduced by this assumption for the two STEREO spacecraft and find that it can be significant, and it can lead to an overestimation of the transient velocity as seen from STEREO-A and an underestimation as seen by STEREO-B. This has implications for the prediction or solar wind transients at $1 \mathrm{AU}$ and hence is important for the study of space weather.
\end{abstract}

Keywords Coronal mass ejections $\cdot$ Corotating interaction regions $\cdot$ Solar wind

\section{Introduction}

\subsection{STEREO}

Launched in 2006, the STEREO mission (Kaiser et al., 2007) observes transients in the solar wind as they propagate from the Sun towards 1 AU, with particular focus on those directed towards the Earth. The mission consists of two near-identical spacecraft that are in Earth-like heliocentric orbits, with one craft (STEREO Ahead, or A) at a radial distance

T.M. Conlon $(\bowtie) \cdot$ S.E. Milan

RSPPG, Department of Physics and Astronomy, University of Leicester, Leicester, LE1 3LY, UK

e-mail: tmc9@leicester.ac.uk

J.A. Davies

RAL Space, STFC Rutherford Appleton Laboratory, Harwell Oxford, Didcott, OX11 0QX, UK 
slightly less than $1 \mathrm{AU}$ and the other (STEREO Behind, or B) at a radial distance slightly greater than $1 \mathrm{AU}$. This difference in orbital distance causes STEREO-A to move at a greater angular velocity and hence to drift ahead of the Earth in its orbit and STEREO-B to move with a slower angular velocity, drifting behind the Earth. The angular separation between each spacecraft and the Sun-Earth line increases by approximately $22.5^{\circ}$ per year.

Each spacecraft carries both in-situ and remote sensing instruments, a total of 16 instruments per craft, split into four instrument packages. This study considers observations by instruments from the Sun-Earth Connection Coronal and Heliospheric Investigation (SECCHI). The SECCHI package on each spacecraft (Howard et al., 2008; Eyles et al., 2009) provides STEREO's remote imaging capabilities. It consists of two coronagraphs (COR1 and COR2), an extreme ultraviolet imager (EUVI) and a pair of heliospheric imagers (HI-1 and HI-2). Each of the coronagraphs and heliospheric imagers observe white light that has been Thomson scattered from electrons in the solar corona and inner heliosphere. Together, the two heliospheric imagers on each spacecraft provide a field of view encompassing $4.0-88.7^{\circ}$ from the spacecraft-Sun line (known as the "elongation" angle, $\epsilon$ ) in the ecliptic plane. Incorporating the coronagraphs and ultraviolet imagers, the field of view comprehensively covers the region of space between the Sun and Earth. While the Earth nominally lies within the field of view, it was occulted near the start of the mission. The separation of the craft gives a stereoscopic view of the region of space between the Sun and Earth, and a variety of techniques have been developed that allow estimates of the trajectory and velocity of a solar wind feature to be made, using both single-spacecraft measurements and simultaneous measurements from the pair of spacecraft. These estimates allow a greater predictive capability of the arrival of solar wind transients at Earth.

Solar wind transients themselves generally fall into one of two categories. Coronal mass ejections (CMEs) occur when an eruptive event launches a large amount of coronal material into the heliosphere, with velocities of $100 \mathrm{~km} \mathrm{~s}^{-1}$ to $1000 \mathrm{~km} \mathrm{~s}^{-1}$. A corotating interaction region (CIR) occurs when sources of fast and slow solar wind are adjacent to each other. Faster solar wind catches up with slower solar wind ahead and we observe a denser interface between these streams. The solar wind source regions corotate with the Sun. The conditions inherent to a CIR are less extreme than those for a CME. For more information on CMEs and CIRs see e.g. Burlaga, Harvey, and Sheeley (2001) and Wang (2005). In this study we will consider the retrieval of direction and velocity information of CMEs and CIRs from SECCHI observations.

\section{J-Maps and Time-Elongation Profile Fitting}

To enhance the visibility of weak intensity features in the raw images, running differenced $\mathrm{HI}$ and COR images from SECCHI are normally used. In sequences of such images, density features can be seen to propagate from near the Sun out through the heliosphere. To emphasise the motion of such transient features, observations along a fixed solar radial from each difference image, are stacked vertically to form a plot of intensity difference versus elongation ( $y$-axis) and time ( $x$-axis). Such time-elongation maps are known as "J-maps". J-maps can be made along any position angle but are most commonly made from ecliptic observations. J-maps can also be made from background subtracted images (e.g. Harrison et al., 2012). For more information on J-maps see Sheeley and Walters (1999) and Davies et al. (2009).

As first proposed by Sheeley and Walters (1999), the variation of elongation with time of a transient (its time-elongation profile), which can be extracted from a J-map, can be used 
Figure 1 The relationship between the locations of STEREO-A and B, the Sun (S) and a plasma blob $(\mathrm{P})$ moving radially with speed $V_{r} . \phi_{\mathrm{A}}$ and $\phi_{\mathrm{B}}$ are the angles between the spacecraft-Sun line and the direction of propagation of $\mathrm{P}$. $\epsilon_{\mathrm{A}}$ and $\epsilon_{\mathrm{B}}$ are the elongation angles, the angles at each spacecraft between $\mathrm{S}$ and $\mathrm{P}$. The arrows show the sense of motion of STEREO-A and -B in their orbit around the Sun.

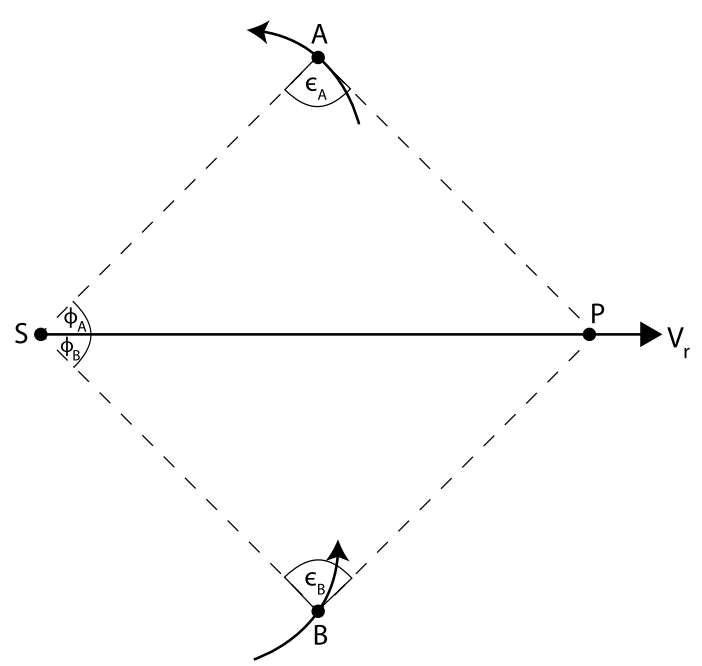

to estimate its radial speed and direction of travel (e.g. Wood, Howard, and Socker, 2010; Liu et al., 2013). These techniques are usually used to determine the motion of transients travelling in the ecliptic plane, although can be applied out of the ecliptic. All of the techniques detailed here apply to single-spacecraft measurements, though there are versions of each technique that apply to stereoscopic spacecraft measurements (Liu et al., 2010; Lugaz, 2010).

\subsection{Fixed- $\phi$ Fitting (FPF)}

In Figure 1, in the heliocentric Earth ecliptic (HEE) coordinate system and looking down on the ecliptic plane, one can consider a particular element of plasma $(\mathrm{P})$ as a point propagating radially out from the Sun (S) with assumed constant radial velocity $V_{r}$. We label the angle between the spacecraft-Sun line and P-S line $\phi_{\mathrm{A}}$ for $\angle \mathrm{ASP}$ and $\phi_{\mathrm{B}}$ for $\angle \mathrm{BSP}$ (for more discussion of the manner in which the angle $\phi$ is defined see Davies et al. (2013)), and the angle between the spacecraft-Sun line and spacecraft-P line as $\epsilon, \epsilon_{\mathrm{A}}$ for $\angle \mathrm{PAS}$ and $\epsilon_{\mathrm{B}}$ for $\angle \mathrm{PBS}$. In this geometry, $\epsilon_{\mathrm{A}}$ is measured East of the Sun and $\epsilon_{\mathrm{B}}$ West. Using the sine rule, it is possible to arrive at the following relationship between the elongation and time,

$$
\epsilon(t)=\arctan \left(\frac{V_{r} t \sin \phi}{r_{\mathrm{sc}}(t)-V_{r} t \cos \phi}\right)
$$

with $t$ the time since launch of the plasma element, $r_{\mathrm{sc}}$ the orbital distance of the observer from the Sun, and $V_{r}$ the radial speed of the plasma point (Rouillard et al., 2008). For a given $\phi$ and $V_{r}$, it is possible to calculate the theoretical variation of elongation with time (i.e. the time-elongation profile) of such a point-like solar wind transient. Therefore, the observed time-elongation profile of a solar wind transient can be used to find the best-fit combination of $V_{r}$ and $\phi$ using a fitting method such as described by Lugaz (2010) to determine the motion of the transient. This technique is most applicable to transients which can be well described by a point particle approximation, i.e. CIR-entrained blobs (e.g. Rouillard et al., 2008) and narrow CMEs. For more discussion of this technique see e.g. Rouillard et al. (2008) and Kahler and Webb (2007). 
Figure 2 The different geometries of the fixed- $\phi$ and harmonic mean fitting techniques (Davies et al., 2012). This diagram follows the notation used by Davies et al. (2012) and Möstl et al. (2011) and uses $d_{0}$ instead of $r_{\mathrm{sc}}$.

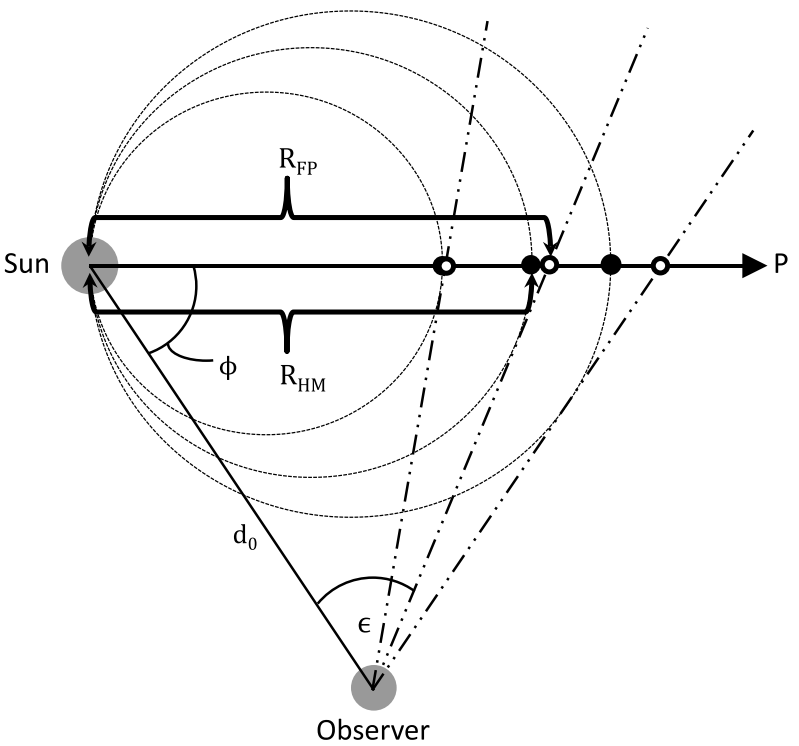

\subsection{Harmonic Mean Fitting (HMF)}

The fixed- $\phi$ technique assumes that the feature is well described by a point. A development of this technique assumes that the feature is more extended in cross section - indeed that it forms an expanding circle with its rear end fixed to Sun centre. Figure 2 (Figure 1a from Davies et al., 2012) illustrates the viewing geometries of both the fixed- $\phi$ and the harmonic mean fitting techniques. It is assumed that the leading edge of the feature observed in a J-map is the tangent to the circle. When this line-of-sight is projected onto the line of motion of the feature (Sun-P line), it leads to a misrepresentation of the actual position of the feature along the Sun-P line. The harmonic mean technique compensates for this misrepresentation by finding the intersection of the circle with the Sun-P line. Then we find that $\epsilon(t)$ can be expressed in the form

$$
\epsilon(t)=\arccos \left(\frac{-b+a \sqrt{a^{2}+b^{2}-1}}{a^{2}+b^{2}}\right)
$$

with $a$ and $b$ defined as

$$
a=\frac{2 r_{\mathrm{sc}}(t)}{V_{r} t}-\cos \phi
$$

and

$$
b=\sin \phi,
$$

which were first written down in the form presented here by Möstl et al. (2011) after Lugaz, Vourlidas, and Roussev (2009).

Davies et al. (2012) found that the two models described so far can be considered as two extremes of a common geometry. They use a more generalised HMF-like analysis, in which the circular CME front is not fixed to the Sun, which they call a self-similar expansion 
(SSE). As with the other techniques, this has also been applied to stereoscopic observations (Davies et al., 2013).

\section{Accounting for Spacecraft Motion}

The FPF, HMF, and SSEF single-spacecraft fitting techniques based on the geometries discussed in the previous section permit $V_{r}$ and $\phi$ to be retrieved from an observed timeelongation profile of a solar wind transient. These techniques assume $\phi$ and $V_{r}$ are constant over the duration of the feature observed. They also assume that the transient's propagation path is fixed with respect to the Sun-spacecraft line during the time for which it is observed. However, this will not be true even if the transient does not deflect away from radial propagation during its travel because the spacecraft themselves are moving in the inertial frame. If we initially consider that the STEREO spacecraft orbit approximately with the Earth, this means that they rotate about the Sun with a period of 1 year. If a transient has a velocity of some $300 \mathrm{~km} \mathrm{~s}^{-1}$, then during the six day travel time of the transient to $1 \mathrm{AU}$ the spacecraft will have moved almost $6^{\circ}$ around its orbit. If we consider specifically the situation in the ecliptic plane, the sense of the orbital motion is shown by the arrows in Figure 1 and this orbital motion means that $\phi$ (the angle of propagation of the transient relative to the Sun-spacecraft line, which, in this case, equates to the ecliptic latitude relative to the Sunspacecraft line) will change with an angular velocity of $\frac{360}{365.25}^{\circ}$ day $^{-1}$ during the outward journey of the transient. This can be combined with the smaller effect from the rate at which the STEREO spacecraft separate from the Sun-Earth line, to arrive at a modified version of Equation (1) for the fixed- $\phi$ geometry, given by

$$
\epsilon(t)=\arctan \left[\frac{V_{r} t \sin \left(\phi_{0} \pm \frac{360 \pm 22.5}{n_{y}} t\right)}{r_{\mathrm{sc}}(t)-V_{r} \cos \left(\phi_{0} \pm \frac{360 \pm 22.5}{n_{y}} t\right)}\right] .
$$

This expression is written assuming that $t$ is in seconds, $n_{y}$ is the number of seconds in a year and $\phi_{0}$ is the $\phi$ value when $\epsilon=0$. Where there is a, \pm+ refers to STEREO-A and refers to STEREO-B. If we apply the same correction to the harmonic mean model, then we arrive at the expressions given in Equations (6) - (8). We have

$$
\epsilon(t)=\arccos \left(\frac{-b+a \sqrt{a^{2}+b^{2}-1}}{a^{2}+b^{2}}\right)
$$

with $a$ and $b$ defined as

$$
a=\frac{2 r_{\mathrm{sc}}}{V_{r} t}-\cos \left(\phi_{0} \pm \frac{360 \pm 22.5}{n_{y}} t\right)
$$

and

$$
b=\sin \left(\phi_{0} \pm \frac{360 \pm 22.5}{n_{y}} t\right) .
$$

Similarly for SSE, $\phi$ is modified in the same way. The correction presented in this section is less easily applied out of the ecliptic, where $\phi$ is no longer the ecliptic longitude relative to the observer and so Equations (5) - (8) are applicable only for transient trajectories in the ecliptic plane, where the spacecraft motion effect is greatest. 

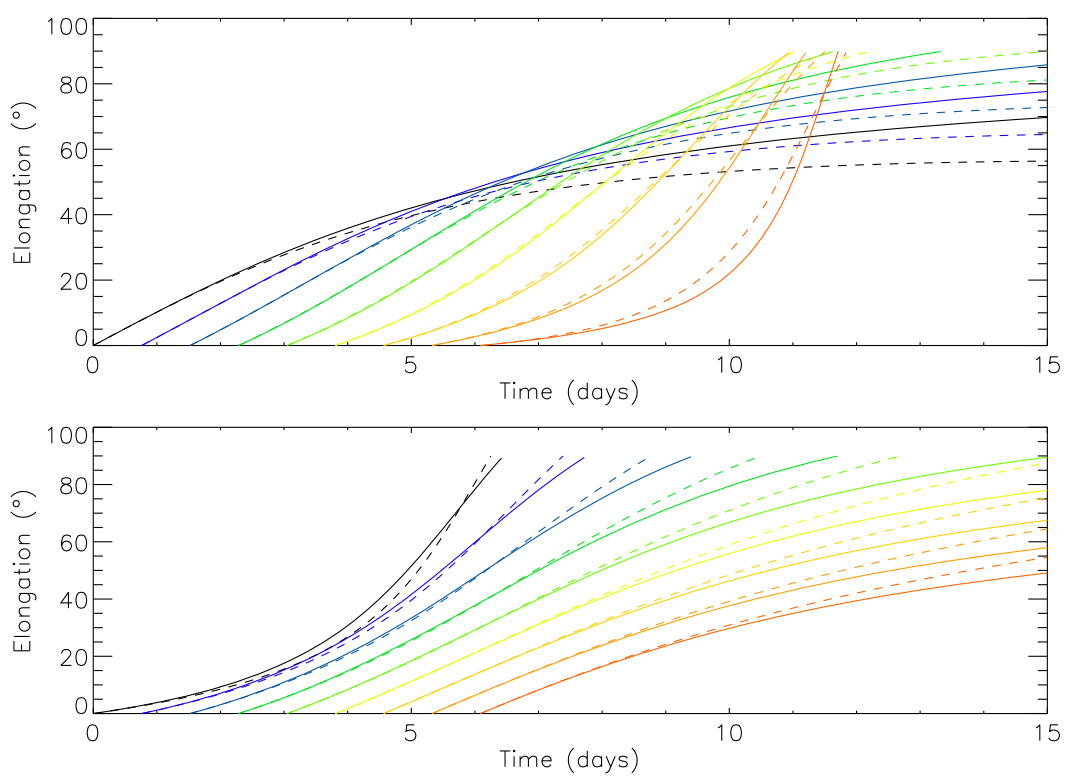

Figure 3 Modelled elongation-time profiles for a series of theoretical transients ejected from the Sun at different values of $\phi_{0}$ and assuming FPF. The solid lines assume constant $\phi$, dotted traces correct for spacecraft motion. The upper panel is for STEREO-A and the lower panel for STEREO-B. Each successive profile is separated by $\approx 16 \mathrm{~h}$ with $\phi$ varying in the range $[20,90]$ in the top panel and $[90,20]$ in the bottom. In each panel, the plots are made using a radial speed of $300 \mathrm{~km} \mathrm{~s}^{-1}$.

In the rest of this paper, we assess the effect of taking spacecraft motion into account when performing fitting, to determine if it makes a significant difference from the values of $V_{r}$ and $\phi$ retrieved from the fitting. In Figure 3, the top panel shows the effect of taking into account spacecraft motion in simulated ecliptic time-elongation profiles as seen from STEREO-A HI and the bottom panel as seen from STEREO-B HI, for the fixed- $\phi$ model. The solid lines correspond to a set of simulated profiles computed with $\phi$ values varying by $10^{\circ}$ from one trace to the next with $\phi_{\mathrm{A}}$ running from $90^{\circ}$ to $20^{\circ}$ in the top panel and $\phi_{\mathrm{B}}$ from $20^{\circ}$ to $90^{\circ}$ in the bottom panel using Equation (1). The dashed lines correspond to that same trace, but including the orbital motion of the spacecraft [i.e. Equation (5)]. Figure 4 shows this for the harmonic mean geometry (Equations (2)-(4) for the solid line and Equations (6) - (8) for the dashed line). In each case, the profiles are separated by $\approx 16 \mathrm{~h}$. These particular traces are for a radial propagation speed of $300 \mathrm{~km} \mathrm{~s}^{-1}$.

Figures 3 and 4 show that including orbital motion of the spacecraft can make an observable difference, up to $\approx 5^{\circ}$ of elongation over a transit time of six days. It would appear, therefore, that including orbital motion of the spacecraft in the single-spacecraft FPF and HMF techniques could make a significant difference from the retrieved speed and direction. We evaluate the effect on the fitted parameters in the next section.

\section{Effect of Including Spacecraft Motion in FPF and HMF}

In this section, we assess the difference in the retrieved parameters that is made by including the effects of the orbital motion of the spacecraft. We simulate time-elongation 

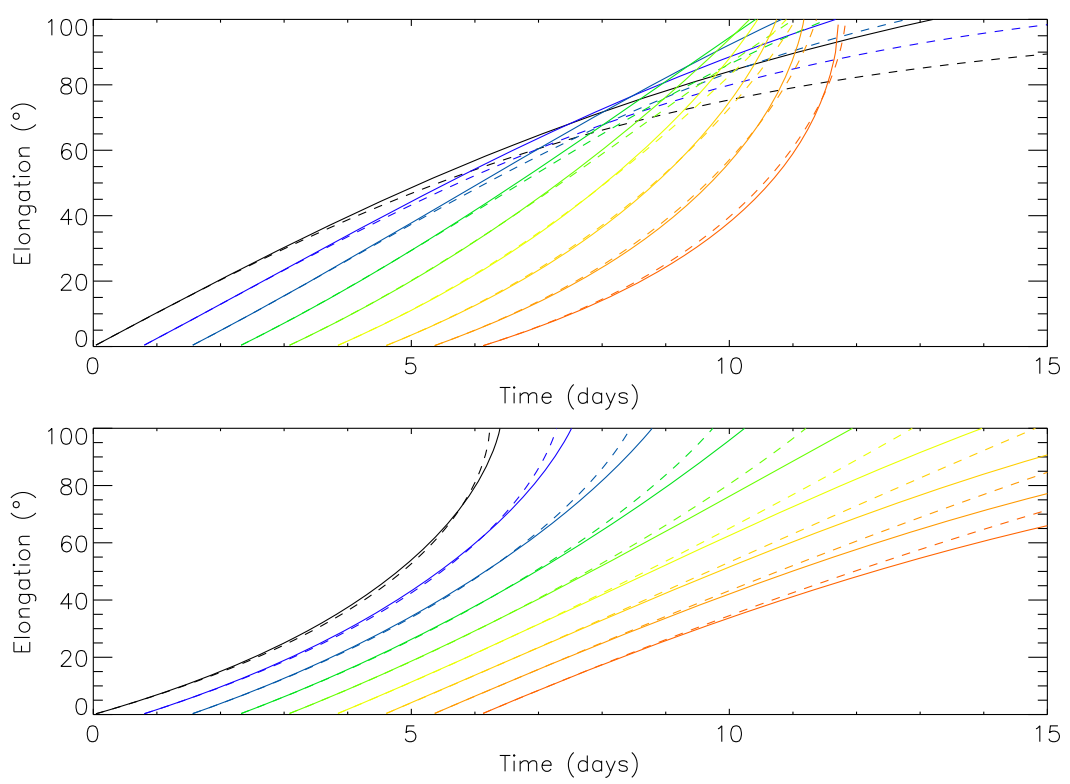

Figure 4 Figure 3 but as shown for the harmonic mean fitting technique.

profiles accounting for spacecraft motion and then perform a best fit to these profiles without accounting for spacecraft motion, i.e. using the technique used in previous studies. In this way it should be possible to assess how much difference this effect makes to the fitting. The simulated time-elongation profiles were created using $10^{\circ} \leq \phi_{0} \leq 170^{\circ}$, $200 \mathrm{~km} \mathrm{~s}^{-1} \leq V_{r} \leq 800 \mathrm{~km} \mathrm{~s}^{-1}$ in increments of $10^{\circ}$ and $50 \mathrm{~km} \mathrm{~s}^{-1}$, respectively, and traced out for 50 1-h time increments. Using a fixed number of time increments ensures that the simulated traces are affected by spacecraft motion for the same length of time and the upper velocity value and the number of time increments were picked to restrict the traces out to radial distances of less than the typical orbital distance of the relevant spacecraft $(\approx 1 \mathrm{AU})$. Figure 5 shows the final radial distance (top panel) and elongation values (bottom four panels) reached by the simulated traces. In the bottom four panels we have the final elongations for STEREO-A using FPF (top left panel), STEREO-B using FPF (bottom left) STEREO-A using HMF (top right) and STEREO-B using HMF. We can see that for the majority of the parameter space investigated, the final elongation value reached is less than $60^{\circ}$. The fitting method itself is that described by Lugaz (2010), fitting $V_{r}$ values of $200-5000 \mathrm{~km} \mathrm{~s}^{-1}$ in $1 \mathrm{~km} \mathrm{~s}^{-1}$ increments and $\phi$ of $-10-190^{\circ}$ in $1^{\circ}$ increments. The traces were also subsequently fitted to a start time of $\pm 5 \mathrm{~h}$ of the launch time, i.e. $\pm 5 \mathrm{~h}$ of the first point of the profile. In Figure 6 we present simulated fixed- $\phi$ (top panel) and HM (bottom panel) time-elongation profiles for STEREO-A assuming that there is:

1) no spacecraft motion over the duration of the profile (blue),

2) accounting for spacecraft motion (black), and

3) the best fit based on fitting the uncorrected equation to the corrected trajectory (red).

The values noted on each panel are the values used to simulate the curves. For comparison, the best-fitted values are $V_{r}=294 \mathrm{~km} \mathrm{~s}^{-1}$ and $\phi=17^{\circ}$ for fixed- $\phi$ fitting and 

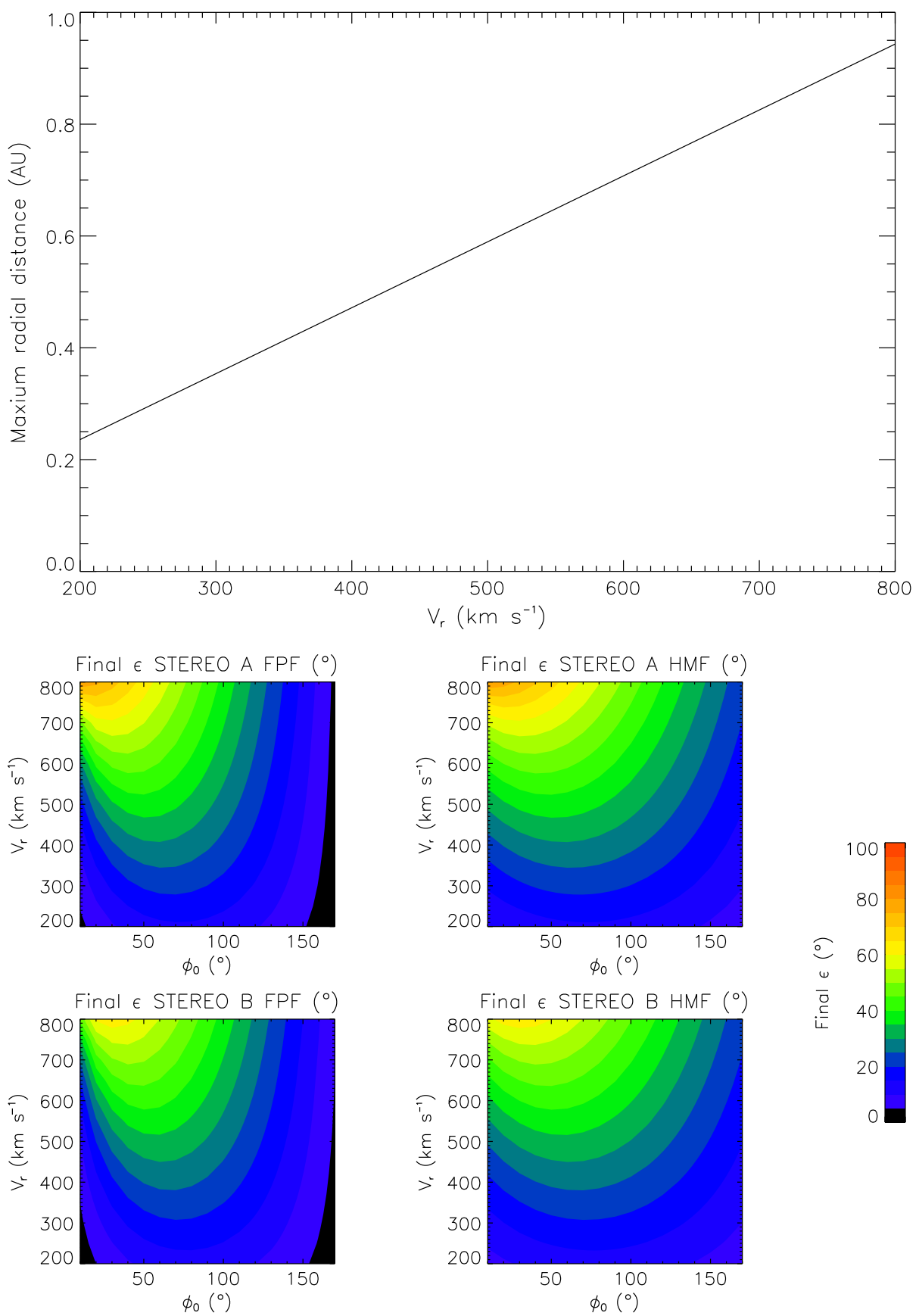

Figure 5 The top panel shows the maximum distance reached by simulated traces across the parameter space of the investigation. In the bottom four panels can be seen the final elongation values reached by the traces across the parameter space for STEREO-A and FPF (top left), STEREO-B and FPF (bottom left), STEREO-A and HMF (top right), and STEREO-B and HMF (bottom right). 
Figure 6 Two panels, in each showing a trace created assuming the inclusion of spacecraft motion (black), neglecting spacecraft motion (blue) and the result from a fit that neglects spacecraft motion to data including spacecraft motion (red). This is done for both the fixed- $\phi$ (top) and the harmonic mean (bottom) techniques, and it models the traces for $501-\mathrm{h}$ time steps.
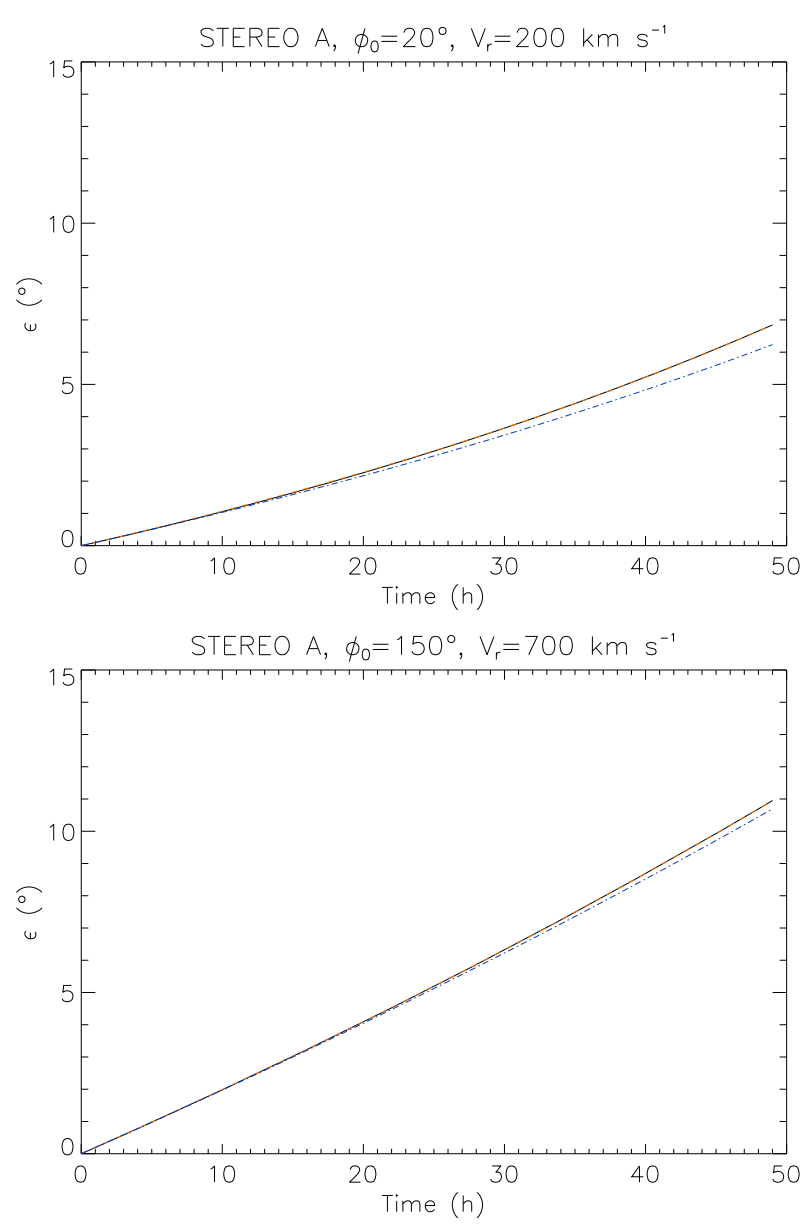

$V_{r}=235 \mathrm{~km} \mathrm{~s}^{-1}$ and $\phi=8^{\circ}$ for harmonic mean fitting. It can be seen in Figure 6 that the fitting process effectively finds a good fit to the corrected profile, even using the uncorrected equation.

In Figure 7, there are four contour plots. These show the difference between the input radial speed and the retrieved radial speed, denoted as $\Delta V_{r}$ and the difference between starting modelled $\phi$ value of a trace $\left(\phi_{0}\right)$ and the best-fit value (i.e. input $\phi$ minus retrieved $\phi$ ), using the fixed- $\phi$ approximation, denoted by $\Delta \phi$. Positive values (red) indicate that the input values are larger and negative values (blue) that the retrieved values are larger.

Considering first the speed plot for STEREO-A (the upper-left panel), we can see that the magnitude of $\Delta V_{r}$ has its largest values for extreme values of $\phi_{0}$, being smaller for more moderate values, and indicates that not accounting for spacecraft motion will lead to an overestimation of the radial speed for large and small $\phi$. Considering the corresponding plot for STEREO-B, the effect is reversed, with an underestimate in radial speed at small and large angles. So there is similar behaviour seen from each of the STEREO spacecraft, but mirrored, so that when the speed is being underestimated in one spacecraft, it is being overestimated by the other. Typically, $\Delta V_{r}$ is a few tens of $\mathrm{km} \mathrm{s}^{-1}$. 

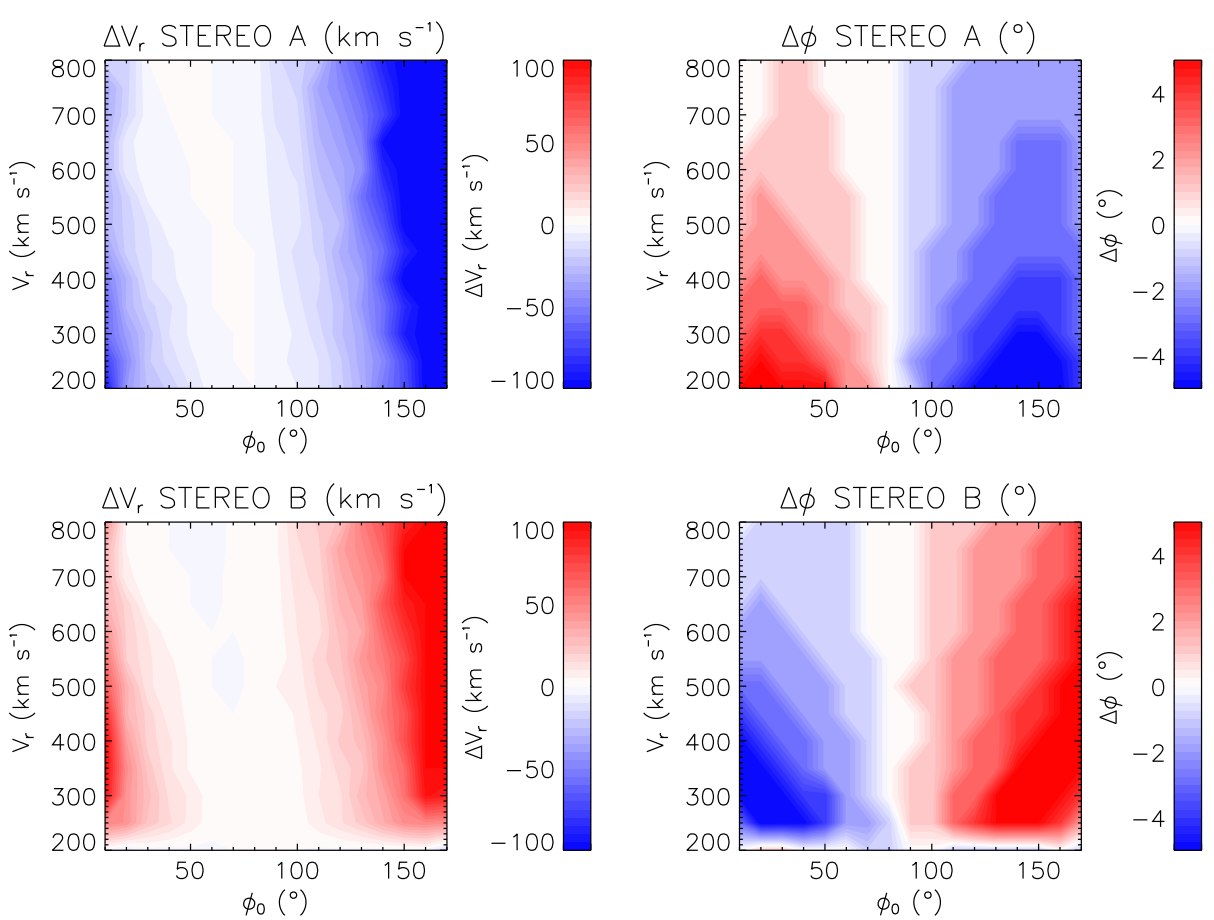

Figure 7 Four contour plots showing the difference made by allowing $\phi$ to vary and then fitting to this using fixed- $\phi$ fitting. The colour scale saturates at red for very large positive values and blue for very large negative values.

Looking now at the right hand two plots, which show the behaviour of $\Delta \phi$ it can be seen that there is an element of symmetry here also. In each case, the magnitude of $\Delta \phi$ is larger for extreme $\phi_{0}$ and smaller radial speeds. If we consider the corresponding plot for STEREO-B (bottom-right panel) then it can be seen that the sense of $\Delta \phi$ effect is reversed. Overall in most regions of parameter space, $\Delta \phi$ is no larger than a few degrees.

In Figure 8, we show similar contour plots for the harmonic mean fitting, with very similar features present as in the previous set of contour plots. Considering each of the plots, however, it can be seen that the size of $\Delta V_{r}$ is larger for fixed- $\phi$ and that of $\Delta \phi$ is larger for the harmonic mean method.

It is perhaps initially surprising here that $\Delta V_{r}$ keeps the same sense for a given spacecraft and only changes sense between spacecraft. If we look at the top panel of Figure 3 then we can see that by eye we might expect the sense of $\Delta V_{r}$ needed to compensate for the spacecraft motion to be opposite when one considers large $v$ s. small values of $\phi_{0}$ (i.e. positive $\Delta V_{r}$ for one extreme of $\phi_{0}$ and negative for the other). To put forward one possible explanation, let us consider a high $\phi$ trace seen by STEREO-A, such as that seen in Figure 9. At early times, an overestimate of $\phi$ leads to an overestimate of $V_{r}$ to compensate and this effect may be enough to offset the effects of spacecraft motion that would otherwise reduce the measured elongation values from what we would expect, an effect that one would expect to lead to an underestimate of $V_{r}$.

As previously mentioned, the traces were simulated for 501 -h time steps. This ensured that the faster traces did not exceed $\approx 1 \mathrm{AU}$ in radial distance. This of course also means that the slower traces were only simulated through the inner heliosphere. In some instances, the 

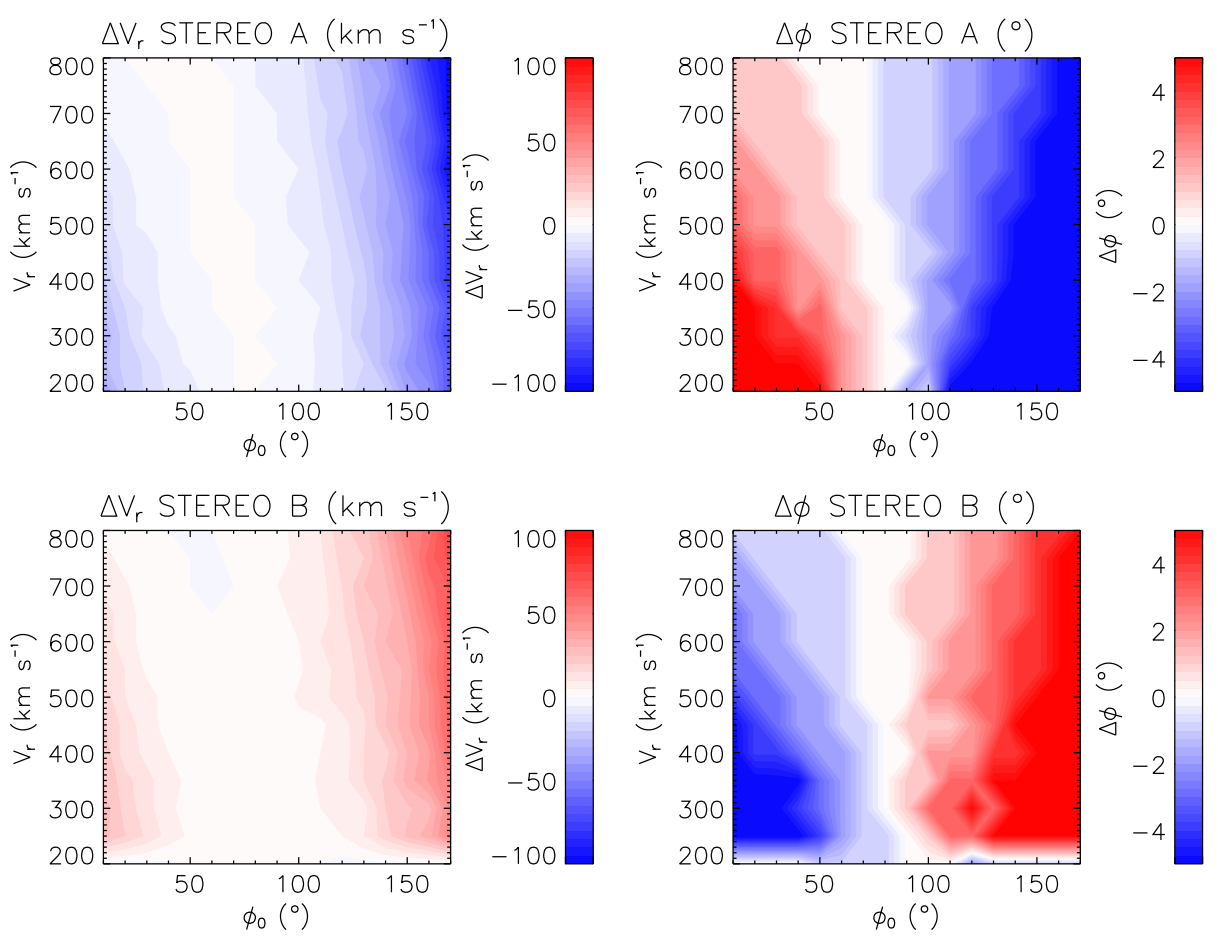

Figure 8 Four contour plots showing the difference made by allow $\phi$ to vary when using harmonic mean fitting. The colour scale saturates at red for very large positive values and blue for very large negative values.

slower traces were separately simulated out to greater radial distances (though still within $1 \mathrm{AU})$ but this was not found to alter the results significantly.

\section{Conclusion}

We have assessed the effect of not accounting for the movement of the STEREO spacecraft when predicting the speed and propagation direction of solar wind transient signatures in the STEREO HI observations, and shown that this can make a noticeable difference from the results, causing the inferred radial speed to differ by tens of $\mathrm{km} \mathrm{s}^{-1}$ or more for extreme viewing geometries. We have assessed the effect for both the fixed- $\phi$ and harmonic mean fitting techniques and found that both are affected by not accounting for this movement, with the radial speed affected more for fixed- $\phi$ and propagation direction affected more for harmonic mean. For either technique, however, it is concluded that the effects of spacecraft motion should be taken into account. There are other sources of error associated with HI observations and some of these could well be larger than the effects from spacecraft motion discussed here (for example inaccuracies due to the assumed geometry of features), though this does not change the fact that spacecraft motion does have a part to play and accounting for this source of error will have direct implications for the predicted arrival times of solar wind transients at Earth and hence for space weather studies, and it will potentially become especially important for planned heliospheric imagers on the upcoming Solar Probe and Solar Orbiter missions, as these orbit closer to the Sun and so the effect could be enhanced. 
Figure 9 Same as Figure 6 but for greater $V_{r}$ and $\phi$.
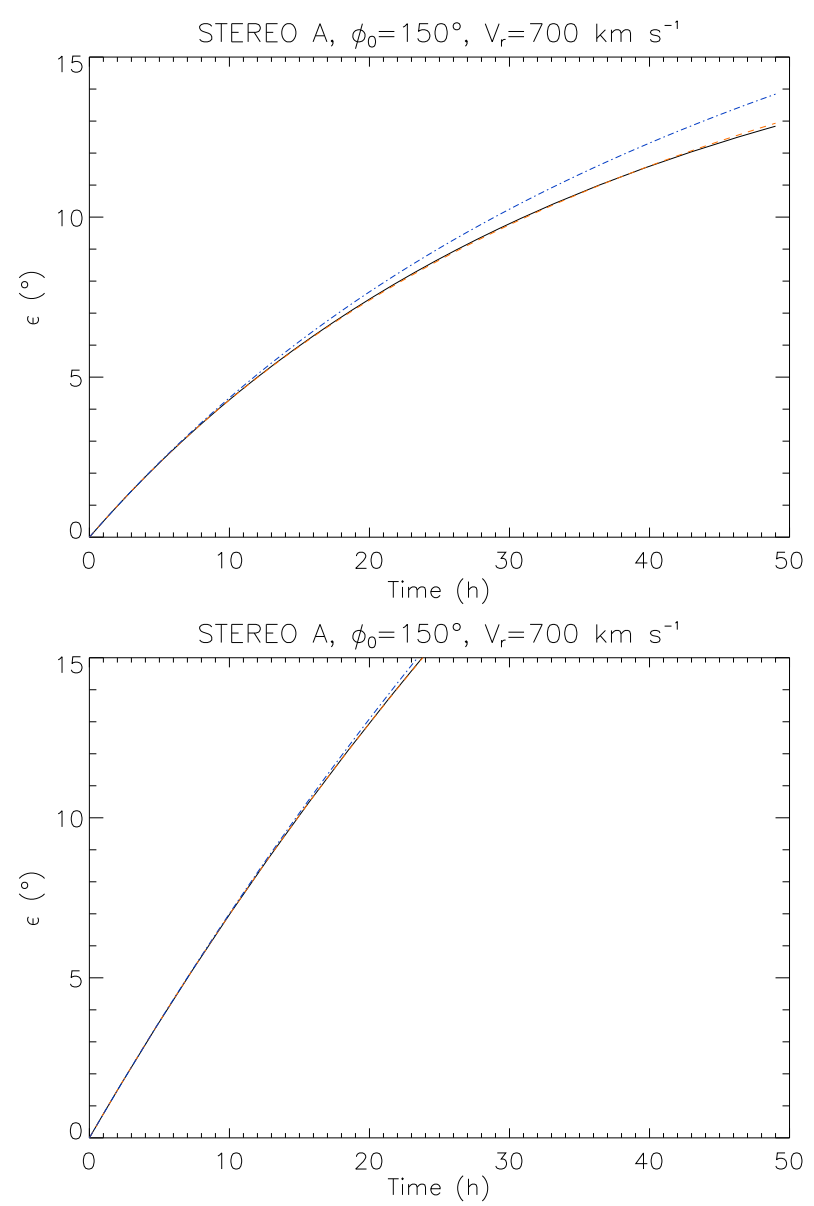

Acknowledgements The authors would like to thank the Science and Technology Facilities Council (STFC) and United Kingdom Space Agency (UKSA) for making this research possible. T.M. Conlon was supported by an STFC, UK, studentship, and S.E. Milan was supported on STFC grant ST/K001000/1.

Open Access This article is distributed under the terms of the Creative Commons Attribution License which permits any use, distribution, and reproduction in any medium, provided the original author(s) and the source are credited.

\section{References}

Burlaga, L.F., Harvey, K.L., Sheeley, N.R.: 2001, A transitory corotating stream, a short-lived coronal hole, and related magnetic fields. J. Geophys. Res. 106, 24915.

Davies, J.A., Harrison, R.A., Rouillard, A.P., Sheeley, N.R., Perry, C.H., Bewsher, D., Davis, C.J., Eyles, C.J., Crothers, S.R., Brown, D.S.: 2009, A synoptic view of solar transient evolution in the inner heliosphere using the heliospheric imagers on STEREO. Geophys. Res. Lett. 36, L02102.

Davies, J.A., Harrison, R.A., Perry, C.H., Möstl, C., Lugaz, N., Rollett, T., et al.: 2012, A self-similar expansion model for use in solar wind transient propagation studies. Astrophys. J. 750, 23.

Davies, J.A., Perry, C.H., Trines, R.M.G.M., Harrison, R.A., Lugaz, N., Möstl, C., Liu, Y.D., Steed, K.: 2013, Establishing a stereoscopic technique for determining the kinematic properties of solar wind transients based on a generalized self-similarly expanding circular geometry. Astrophys. J. 777, 167. 
Eyles, C.J., Harrison, R.A., Davis, C.J., Waltham, N.R., Shaughnessy, B.M., Mapson-Menard, H.C.A., et al.: 2009, The heliospheric imagers onboard the STEREO mission. Solar Phys. 254, 387.

Harrison, R.A., Davies, J.A., Möstl, C., Liu, Y., Temmer, M., Bisi, M.M., et al.: 2012, An analysis of the origin and propagation of the multiple coronal mass ejections of 2010 August 1. Astrophys. J. 750, 45.

Howard, R.A., Moses, J.D., Vourlidas, A., Newmark, J.S., Socker, D.G., Plunkett, S.P., et al.: 2008, Sun Earth Connection Coronal and Heliospheric Investigation (SECCHI). Space Sci. Rev. 136, 67.

Kahler, S.W., Webb, D.F.: 2007, V arc interplanetary coronal mass ejections observed with the solar mass ejection imager. J. Geophys. Res. 112, A09103.

Kaiser, M.L., Kucera, T.A., Davila, J.M., Cyr, O.C., Guhathakurta, M., Christian, E.: 2007, The STEREO mission: An introduction. Space Sci. Rev. 136, 5.

Liu, Y., Davies, J.A., Luhmann, J.G., Vourlidas, A., Bale, S.D., Lin, R.P.: 2010, Geometric triangulation of imaging observations to track coronal mass ejections continuously out to 1 AU. Astrophys. J. Lett. $\mathbf{7 1 0}$, L82.

Liu, Y.D., Luhmann, J.G., Lugaz, N., Möstl, C., Davies, J.A., Bale, S.D., Lin, R.P.: 2013, On Sun-to-Earth propagation of coronal mass ejections. Astrophys. J. 769, 45.

Lugaz, N.: 2010, Accuracy and limitations of fitting and stereoscopic methods to determine the direction of coronal mass ejections from heliospheric imagers observations. Solar Phys. 267, 411.

Lugaz, N., Vourlidas, A., Roussev, I.I.: 2009, Deriving the radial distances of wide coronal mass ejections from elongation measurements in the heliosphere - Application to CME-CME interaction. Ann. Geophys. 27, 3479.

Möstl, C., Rollett, T., Lugaz, N., Farrugia, C.J., Davies, J.A., Temmer, M., et al.: 2011, Arrival time calculation for interplanetary coronal mass ejections with circular fronts and application to STEREO observations of the 2009 February 13 eruption. Astrophys. J. 741, 34.

Rouillard, A.P., Davies, J.A., Forsyth, R.J., Rees, A., Davis, C.J., Harrison, R.A., et al.: 2008, First imaging of corotating interaction regions using the STEREO spacecraft. Geophys. Res. Lett. 35, L10110.

Sheeley, N.R., Walters, J.H.: 1999, Continuous tracking of coronal outflows: Two kinds of coronal mass ejections. J. Geophys. Res. 104, 24739.

Wang, C.: 2005, Characteristics of the interplanetary coronal mass ejections in the heliosphere between 0.3 and 5.4 AU. J. Geophys. Res. 110, A10107.

Wood, B.E., Howard, R.A., Socker, D.G.: 2010, Reconstructing the morphology of an evolving coronal mass ejection. Astrophys. J. 715, 1524. 